\title{
12 Positive Change and Quality of Time in Daily Life
}

\begin{abstract}
This chapter focuses on the relationship between "quality" and "time" and how individuals define "time well spent" and use it to bring about optimal experiences in their personal and professional lives. In describing our findings, we report on both qualitative and quantitative data collected over five years, from individuals in seven different countries. At the end of the chapter, we discuss how we are using our findings to create applications of our work, as well as some general lessons about how we as individuals might incorporate these findings into our own lives.
\end{abstract}

Keywords: Time; Quality; Optimal experience; Applications; Lessons.

\subsection{Introduction}

The phrase "Wish not so much to live long as to live well" can be found in the Poor Richard's Almanac, compiled by Benjamin Franklin more than 250 years ago. Interestingly, many of the phrases in this document relate to the theme of time. This particular phrase raises an interesting question: is it better to live long, or live well? What does it mean to live well? What is a "quality life?"

In 2008, the Good Project ${ }^{6}$ launched a study to explore the concept of Quality ${ }^{7}$. We were curious about the meaning of quality, how people judge quality, and if modern technology influences how people think about quality. In other words, does the ability to do things quicker or with more information change the notion of quality? In our study, we sought to understand how individuals with unprecedented access to resources and efficiencies think about the quality of personal belongings, consumer experiences, work, and time. By asking what quality looks like, when it matters, and what experiences shape how people characterize excellence, we wanted to understand the meaning and value individuals in contemporary societies attribute to quality.

When we first began our research, we predicted that individuals would define quality in terms of objects and services. We thought that individuals would list criteria or identify specific features that marked or defined quality. For example, individuals might tell us about a watch that is "high quality" because of its price, the status of the

6 For more information, see www.thegoodproject.org

7 We appreciate and acknowledge the generosity of Faber-Castell which has funded this research. 
store from which it was purchased, the country in which it was made, or the specific features which deem the object "excellent.” Based on our own research on the Good Project, which studied the kind of work that individuals do and how they go about doing it, we wondered if individuals might define quality in terms of their workin terms of "excellence" in their respective professions. However, after interviewing nearly a hundred individuals and surveying thousands of people across the globe, we were surprised and interested to find that "time" was the most unifying concept of quality; indeed "quality time" is how individuals define and judge quality.

Interestingly, "quality time" has become a frequently used phrase in contemporary society. Most often, it is utilized when referring to time spent with others - time to deeply connect with friends or relatives (more than a quick phone call or email). But in order to understand the relationship between "quality" and "time" and how individuals make decisions about the role of "quality time" in their lives, we set out to specifically collect data about these questions. After careful analyses, we coined the term "time well spent" to describe the kind of activities individuals deem worthy of their personal and professional investments of time. In the following chapter, we describe individuals' perceptions of "time well spent" as well as "wasted time," and how individuals bring about optimal experiences through their own meanings of these terms. In describing our findings, we allude to both qualitative and quantitative data collected over five years, from individuals in seven different countries. At the end of the chapter, we discuss how we are using our findings to create applications of our work, as well as some general lessons about how we as individuals might incorporate these findings into our own personal and professional lives. Ultimately, we hope to help people live well and long, but to begin with, we explore what "living well" actually means.

\subsection{Literature Review}

When we began our research in 2008, we conducted an initial review of literature on quality within the business sector ${ }^{8}$. We focused on this literature with the assumption that the business world had spent considerable effort defining and refining the concept. Our assumption proved accurate: we were able to identify trends and perspectives on quality, and reveal valuable characteristics that determine quality goods and services. This brief survey of literature focused on three particular sectors of business: banking, health care, and tourism. Below, we summarize the most relevant understandings for the purposes of this chapter.

Characteristics of quality have evolved steadily during the time that western societies have evolved from a focus on industry, through the service and knowledge

8 Colleague Kathleen Farrell conducted this initial literature review. 
sectors, and adjusted to consider the advent of the Internet. Although numerous perspectives on quality emerged over these years of history, five well-established orientations emerged including: 1) the transcendent or innate character of a product that defies exact definition; 2) a product-based orientation that focuses on difference in elements or attributes; 3) a user-based orientation that assumes that the judgment of quality is determined by the consumer; 4) a manufacturing-based orientation that focuses on level of conformity to production, safety and design standards; and 5) a value-based orientation which uses other constructs such as excellence and consumer trends to determine quality. This final construct is considered in conjunction with cost and marketability.

Garvin (1985) proposed eight dimensions of quality that bridge the above quality dimensions: performance, features, reliability, conformance, durability, serviceability, aesthetics and perceived quality. With the rise of a service-based economy, there also arose a need to redefine quality within this context. Parasuraman, Zeithamel, and Berry (1985) conducted research identifying further characteristics of quality services and later developed an instrument (SERVQUAL) that is still a standard for service quality research (Parasuraman, Zeithamel, and Berry, 1988). These dimensions of service quality include: reliability, responsiveness, competence, access, courtesy, communication, and credibility. With the advent of the Internet, the applicability of these models was once again questioned and further refined. Although many characteristics of in-person service might also be applied to Internet service, there were additional issues to consider such as privacy, accuracy, and availability (to name just a few).

Critiques of the various models surveyed within each of three business realms (banking, health care and tourism) suggest that the business literature provided an important but not complete introduction to how individuals consider quality in their lives. In particular, this initial review suggests that further research into "consumer culture" may help to inform our understanding of quality in important ways. Objects may help us to find meaning in our lives and hone our identities. Additionally, goods and services may be experiences that educate and define us, for example by creating memories (Csikszentmihalyi \& Rochberg-Halton, 1981; Gabriel \& Lang, 1995; Thomson, 2000; Turkel, 2007). These and other such sources might provide a useful framework, moving thinking about quality from a "user-based" perspective to a "human-based" perspective. As we consider the meaning of quality within individuals' lives, this perspective becomes invaluable. As a result, more recently we have focused our review of literature on this "human-based" perspective, and considerations of quality in relation to our understanding of experiences (quality of life, quality of work) and quality of time.

A number of studies examine how individuals prioritize and spend their time and consider the implications on quality of life. For example, Masuda and Sortheix conducted a seven-year longitudinal study of two hundred and two MBA students from 6 different universities in the United States. Participants were surveyed about their 
family, work and leisure goals, family and work values, as well as life satisfaction. When family goals are given priority over work and leisure goals, higher life satisfaction is reported (2011). The implications of this study are important for the public discourse about balance between work, family and leisure, offering empirical evidence for the value of giving individuals more time for their families.

In addition, Mezzich et al. (2011) present an instrument designed to measure quality of life: the Multicultural Quality of Life Index (MQLI). After an extensive review of health care and social science literature, 10 dimensions of quality of life were identified, including Physical Well-being, Psychological/Emotional Well-being, Self-Care and Independent Functioning, Occupational Functioning, Interpersonal Functioning, Social-Emotional Support, Community and Services Support, Personal Fulfillment, Spiritual Fulfillment, and Global Perception of Quality of Life. This instrument was found to be largely successful in terms of ease of use, cultural applicability, comprehensiveness, and self-ratedness. Its ease of use (can be completed in 2-3 minutes) and comprehensiveness indicates that it could have wide applicability in a variety of settings.

Two studies in particular, described below, examine subjective understandings of time and are of particular relevance to considerations of time well spent. Both studies describe the concept of time "affluence." The first of these describes how, counter intuitively, "giving time gets you time." The second of these studies focuses on morality, and investigates whether a focus on giving time makes individuals more ethical.

First, Mogliner et al. (2012) describe subjective and objective uses of time. Whereas objective use of time is limited ( 24 hours in every day, 60 minutes in an hour), subjective use of time is more malleable. Interestingly, our separate experiments suggest that giving of one's time in a prosocial manner increases the subjective understanding of time. This subjective understanding is directly related to one's sense of "selfefficacy" - how effective and useful one feels about oneself. In other words, time affluence can be increased, "Spending time on others makes people feel like they have done a lot with their time - and the more they feel they have done with their time, the more time they will feel they have.” (p. 1) The authors compare spending time on others versus wasted time, spending time on oneself, or gaining some unexpected free time. Among other things, Mogilner et al. questioned whether spending time on others is experienced as more "meaningful" - considering that deeply meaningful tasks are sometimes characterized as "flow" experiences (Csikszentmihalyi, 1990). The results reveal that time given to another was not reported as more meaningful that time spent on oneself. This and a variety of other factors suggest that only "self-efficacy" explains the effect of giving time on time affluence. The authors also point out that giving time increases not only subjective time, but in some cases can also increase objective time - if the time given to help someone else saves them time on a particular task, for example, helping to work on a house renovation (2012). This study is significant because it suggests that if people spend more time on others, they may feel less time constrained and more effective. This is positive news in the face of 
numerous reports about negative effects of "time famine," or the feeling of not having enough time (deGraaf, 2003; Kasser \& Sheldon, 2009).

In a later, second study, Gino and Mogilner (2013) conducted a series of experiments that examined whether a focus on time, as opposed to money, would make people behave more ethically. Operating with the supposition that those who are focused on money behave in more self-interested and less self-reflective ways, they sought to discover if a focus on time would encourage more self-reflection, and thereby, more ethical behavior. These four different experiments, conducted with varied "primes," tasks and measures, the authors consistently found that moving the focus to time over money decreases the occurrence of dishonesty. These studies are significant for our purposes in that they provide evidence that how people spend their time is related to how they understand themselves.

Finally, we offer a brief look into three additional perspectives to offer a sampling of the current academic discourse around time, which seems to be emerging as a "popular" topic. First, Philosopher Mark Kingwell approaches the issue of time via philosophical discussion about how we spend our leisure time. Using the (only somewhat) hypothetical proposition of a robot working class (although we're not quite there yet, in the future there may well be robot butlers and/or maids) Kingwell considers the consequences of creating such a class of workers. What would we all do with our additional free time? Kingwell distinguishes between the "slacker" and the "idler." The "slacker" is someone who avoids work without a commitment to anything else, whose decisions are dominated by the idea of work as the thing to be avoided. The "idler" is someone not interested in work at all, but committed to something else, which is usually some kind of leisure activity. Through a discussion of economists, philosophers, and thinkers such as Thorstein Veblen, Karl Marx, and Pierre Bourdieu, Kingwell explains that the hypothetical liberation that such technological advancements could create, would in fact only free us to try to outdo one another, become consumers and experience "leisure time that must be filled with experiences supplied by the culture industry.” (p. 7) If individuals are freed from mundane tasks, to acquire more time for leisure, this free time will be occupied by choices made from a different industry - the culture industry. As Kingwell explains it, redistributing work to a robot class is worrisome not because of the effect on the quality of the work but the impact on the quality of ourselves, because "work hones skills, challenges cognition, and at its best, serves noble ends." (p. 8) With this contrast in mind, idle time is much more worthwhile than slacking or wasting time. In other words, how we spend our leisure time changes the quality of both our work and leisure experiences.

Second, in a provocative essay, "In Praise of Laziness" economist and political scientist Joseph Schumpeter (2013) argues for the importance of leisure time. Schumpeter paints a picture of the business world as too frenzied, filled with too many disruptions. In particular, he focuses on creative workers and argues that they require large portions of unbroken time. This description of the overscheduled nature of today's working world is a regular part of the public discourse. Schumpeter's 
perspective suggests that our economy may suffer as creative individuals - who make change - produce a different quality of work.

Third, in Locust and the Bee, Mulgan discusses the relationship between time and value, explaining that individuals' relationship to time has impact on how we interact with those around us. Specifically, he discusses the history and future of capitalism, and considers fields such as healthcare, education and green industry. Considering time as another type of currency, he explains, "For anyone living rationally and wisely, the quality of life will always be a higher value than money or consumption" (224).

In summary, public and academic discourse is replete with the subject of time, and increasingly, concerned with how the quality of our life experiences (both work and leisure) has been impacted with the evolving nature of how we experience time. As discussed below, our study of quality and "time well spent" contributes to this discourse as well as providing suggested applications to help and encourage individuals to structure time in order to allow for "optimal experiences" in their lives.

\subsection{Findings: Our Study}

Between 2008 and 2013, we embarked a large-scale study on "quality," involving participants from around the world, using both quantitative and qualitative measures ${ }^{9}$. Specifically, we conducted in-depth interviews with individuals in several different countries and disseminated a survey to 7000 individuals in seven different countries. We sought to understand the ways in which people define quality, when and how they experience it, and how they make decisions about quality, both in terms of establishing priorities and navigating compromises. At the beginning stages of research, we carried out three rounds of interviews with children, adolescents, and adults in the United States, each one building upon findings from the previous stages. In this way, we were able to refine our research instruments using words and examples that emerged organically in conversations with others. Then, based on our qualitative findings, we developed a comprehensive 74-question survey for adults, which included questions about quality as it relates to objects, work, time, as well as questions about the relative quality of digital and non-digital experiences. The question-

9 Specific countries include: Brazil, China, Germany, India, Indonesia, Turkey, and the United States 
naire was adapted, translated, and disseminated in seven different countries over the period of two years. ${ }^{10}$

\subsubsection{Methodology}

The research on quality was carried out over five years (2008-2013) in four phases. Each phase built on previous findings, including measures and tools of research as well as type of participant (age and locale). Here, we outline the specific phases and our methods used to carry out the work:

\subsubsection{Phase 1}

We conducted 81 in-depth interviews with people in the United States. This phase included three different groups: 1) interviews with 22 participants (based on personal connections) including children, young adults, and adults about the various tools they use in their work and whether the quality of these tools had an impact on the quality of what they produce; 2) based on a "snowball" method, we interviewed 22 adults to understand how individuals define quality in general; and 3) we used Craig's List ${ }^{11}$ in Boston and Seattle to recruit 37 individuals to participate in an interview about their general perspectives on quality, but also more questions about time and how it factors into individuals' overall conceptions of quality. In this phase, all interviews were conducted in-person or over the telephone with semi-structured interview questionnaires. Each interviews lasted about an hour. They were recorded, and the interviewer wrote summary sheets about each interview, including quotes taken down during the actual interview and checked on the recording.

\subsubsection{Phase 2}

We used the themes from our qualitative findings from the first phase to develop a comprehensive Internet survey that was administered to a broader population in the United States. The survey took approximately 20 minutes to complete and included questions about quality as it relates to objects, time, and work, as well as questions about the relative quality of digital and non-digital experiences. To recruit partici-

\footnotetext{
10 There are some limitations to the data collected, primarily that the survey was disseminated through the Internet, therefore individuals had to have access to the Internet in order to complete the survey (which does not always accurately reflect the demography of each country). However, we worked with a consumer awards program, ResearchNow, to recruit the most reflective group as possible in terms of demography. Every person who completed the survey was 18 years of age or older and was a member of the awards program.
}

11 Craig's List is a website that posts classified advertisements. 
pants, we used a combination of Craigslist, Facebook, and an online market research firm called ResearchNow ${ }^{12}$. We secured a "random" sample of 1000 individuals, representative of the United States population in terms of educational attainment, age, employment status, race/ethnicity, and household income.

\subsubsection{Phase 3}

We expanded our research to the global context - incorporating six other counties, including Brazil, China, Germany, India, Indonesia, and Turkey. After some adapting and translating (when appropriate), we continued to work with ResearchNow to secure responses from 1000 individuals in each country whom reflected the demographics of each country (as much as possible given that this was a survey that only could be accessed via the Internet). After a thorough analysis of the data, we met with a group of "country experts," two individuals from each country, who helped us to interpret and make sense of some of the findings in order to double and triple check that we put the findings into appropriate context.

\subsubsection{Phase 4}

We interviewed 27 global employees of Faber Castell to "pilot test" and explore our findings about "time well spent." The participants were located in various countries worldwide. The employees represented different departments within the company (e.g. human resources, sales, marketing, management, etc.) who held various roles within these departments and markets. All interviews were conducted via telephone, and each lasted an hour (and were recorded).

\subsection{Findings}

\subsubsection{Time as an Indicator of Quality}

Though we began our study with key questions about quality in general, we found that "time" is an essential factor in how people define and think about quality in their lives. Even though we sought to understand the relationship between time and quality from the outset of our study, we did not anticipate that the concept of time would be so important to how people make decisions about quality in their personal and professional lives. In our first set of interviews with individuals in the United

12 ResearchNow invites individuals who are affiliated with consumer rewards programs to join their response panel and then draws purposeful samples from the panel to meet client-specific needs (e.g. recruiting a sample that reflects the demography of a specific country). 
States we observed dissonance in people's descriptions of quality. Despite expressing a strong commitment to quality and articulating detailed accounts of what quality looks like, most interviewees also acknowledge that they frequently settle for less than they think is best. When asked about this, they typically explain that although buying high-quality goods and supporting excellent companies is extremely important, ultimately they act in ways that preserve quality time. Rarely did participants talk about the scarcity of time; rather, they explained that when forced to choose between getting coffee at a preferred local shop or at a national chain, they would compromise on their preference if that meant they could spend that time with friends and family. Therefore, although the quality of objects, work, and services matter to people, the quality of time is uniquely important. A female law student, for example, comments, "Quality is something that is worth the time and effort you put in, an object or experience that gives you high return emotionally and spiritually (as opposed to monetarily)." In other words, time is a currency that can ensure life-balance and enable individuals to pursue meaningful relationships and interests.

Interestingly, the survey participants echoed the sentiments of the interviewees: Time is paramount in terms of quality. Specifically, as shown in Figure 1, when asked in what ways quality is most important, Western cultures generally indicated "time” and Eastern cultures indicated "objects”. However, respondents of the survey indicated that objects are considered to be quality for their durability (objects last a long time) and utility (objects function well and help individuals be efficient and productive), which both relate to time - they allow individuals to do the things they like without wasting time or resources (see Figure 2). In other words, individuals ultimately pursue goods based on the intrinsic temporal value that those goods hold (e.g. "Can this help me spend my time better?") rather than questions about the absolute quality of the object itself.

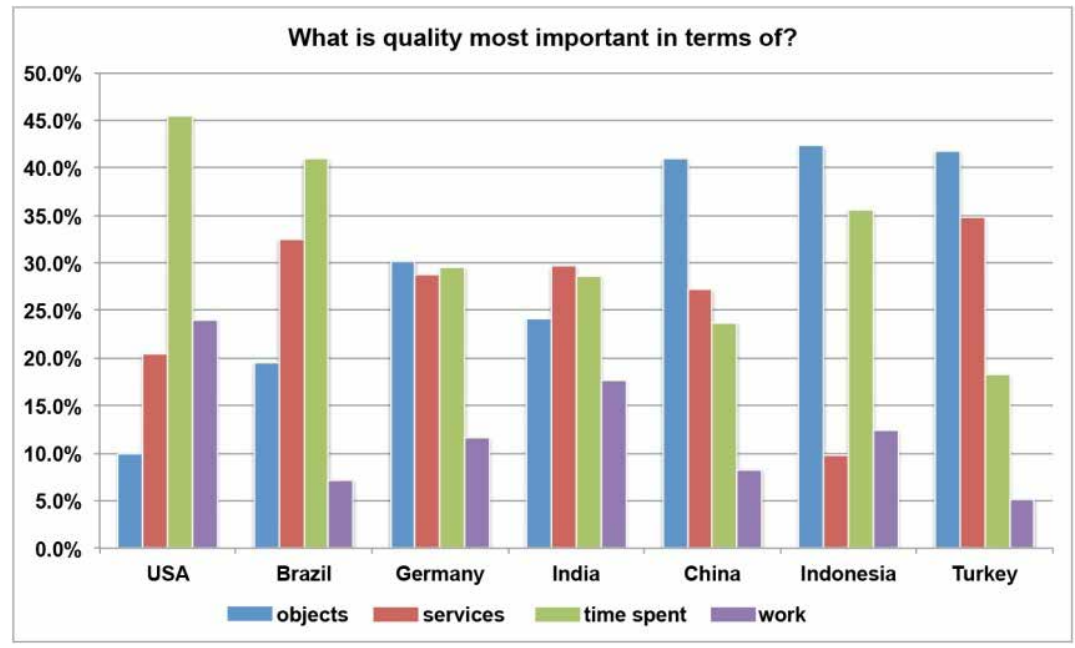

Figure 12.1: What is quality most important in term of? 


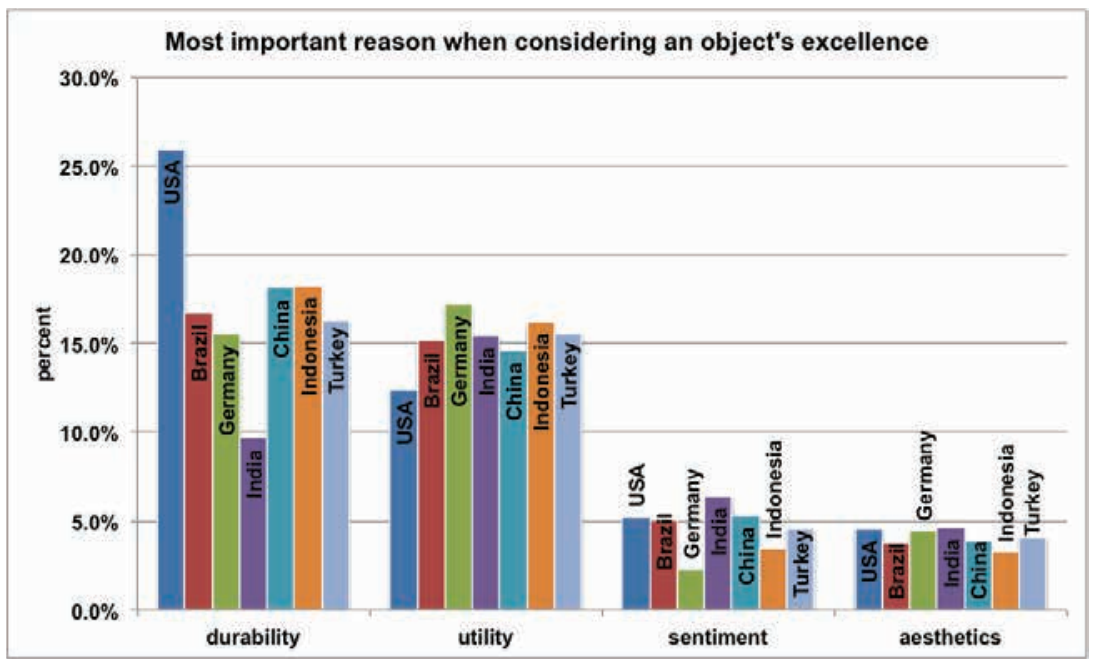

Figure 12.2: Most important reason when condsidering an object's excellence

\subsubsection{Time Well Spent and Wasted Time}

Based on these data, we developed the term "time well spent" to explain the ways in which individuals describe valuable investments and uses of their time. Several individuals indicated that in modern society, how we spend our time has become paradoxically more important as we have accrued more ways in which to spend it. Throughout history, leisure time has been scarce, but never before have we had as many ways to fill our leisure time as we do today. Hunter-gatherers could rest or socialize with others in their society; today, we have myriad ways to spend our leisure time. Based on our survey data, individuals indicated that it can be difficult to choose between spending time with friends, learning new things, watching television, using the Internet, and other things as well. Though in general, individuals feel that "time well spent" includes a variety of activities, the highest ranked items are: spending time with friends and family (19\%), using technology for entertainment (13\%), learning something new (13\%), and hobbies (12\%). Interestingly, in terms of defining "time well spent," activities often carried out by oneself, without contact with others are ranked the lowest of the group: reading, sleeping, and exercising (less than 1\%) and being alone (2\%).

Specifically, we note some differences by country, particularly in terms of how countries' responses related to the Human Development Index (HDI) ${ }^{13}$. Countries with a higher HDI (those above 0.80, namely the United States and Germany) rank

13 The Human Development Index is a composite of the country's social and economic standing. 
family and friends as the top response in terms of defining time well spent (33\% for the United States, $27 \%$ for Germany). Not only are the rankings of family and friends lower for each of the countries with a lower HDI (Brazil, China, Indonesia, India, Turkey), but calling the use of technology for entertainment "time well spent" is higher (Brazil: 12\%, India: 12\%, China: 19\%, Indonesia: 22\%, and Turkey: 11\%; compared to the high-HDI countries of the US [6\%] and Germany [7\%]).

Interestingly, even survey participants who expressed technology for entertainment as "time well spent" also indicated that technology can be "time wasted" - time spent that did not improve the quality of their daily experience. Specifically, technology for entertainment is the highest ranked "time waste" for every country, regardless of the HDI index, though individuals in countries with the highest HDI are more decisive about technology as a "time sink." Seen in Figure 3, the highest HDI countries (United States \& Germany) are much more likely to call technology "time wasted" than the lower HDI countries. However, as is largely evident, more people in most countries believe that technology is more of a "time waste," than "time well spent."

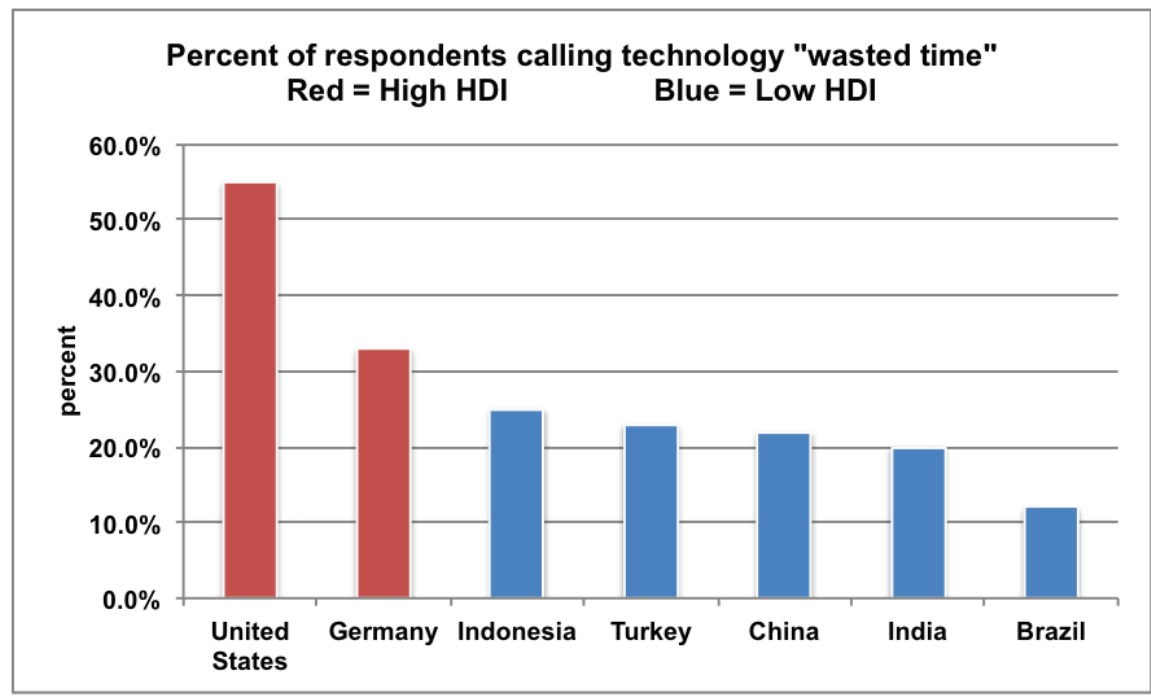

Figure 12.3: Percent of respondents calling technology „wasted time“

This finding shows that despite prevalence of the use of technology, it is a "doubleedged sword" for many individuals. Through both the interviews and surveys, we found that the widespread use of modern technologies (e.g. laptop computers, smartphones, tablets) seems to have both a positive and negative impact on how individuals spend their time. As revealed in our interviews in the United States, individuals praise the ability of technology to keep them connected to friends and family (e.g. via email or Skype, or other venues). However, "surfing the web," or playing games that do not necessarily keep them connected to others, is considered to be a waste 
of time. Moreover, many individuals indicate that technology can lead them to communicate with others inefficiently or impersonally; for example people may be more likely to send an email to a colleague across the hall rather than having a face-to-face conversation.

Perhaps the most revealing data is about how individuals indicated using time within the last 24 hours - in terms of time well spent and wasted time - and how they plan to use future free time. Specifically, at least $20 \%$ of the sample in each of the seven countries surveyed is aware of the fact that they waste their time with technology, and yet do not plan on changing their future behaviors so as not to waste time in that way again. Furthermore, this finding is represented by individuals from countries with varying HDIs, across all ages, and ranges of socioeconomic status, and is equally distributed between males and females (Figure 4). Technology, therefore, presents a unique challenge for many people: it can provide an efficient and unique way to stay connected to others and spend time well, and it can just as easily devolve into a waste of quality time.

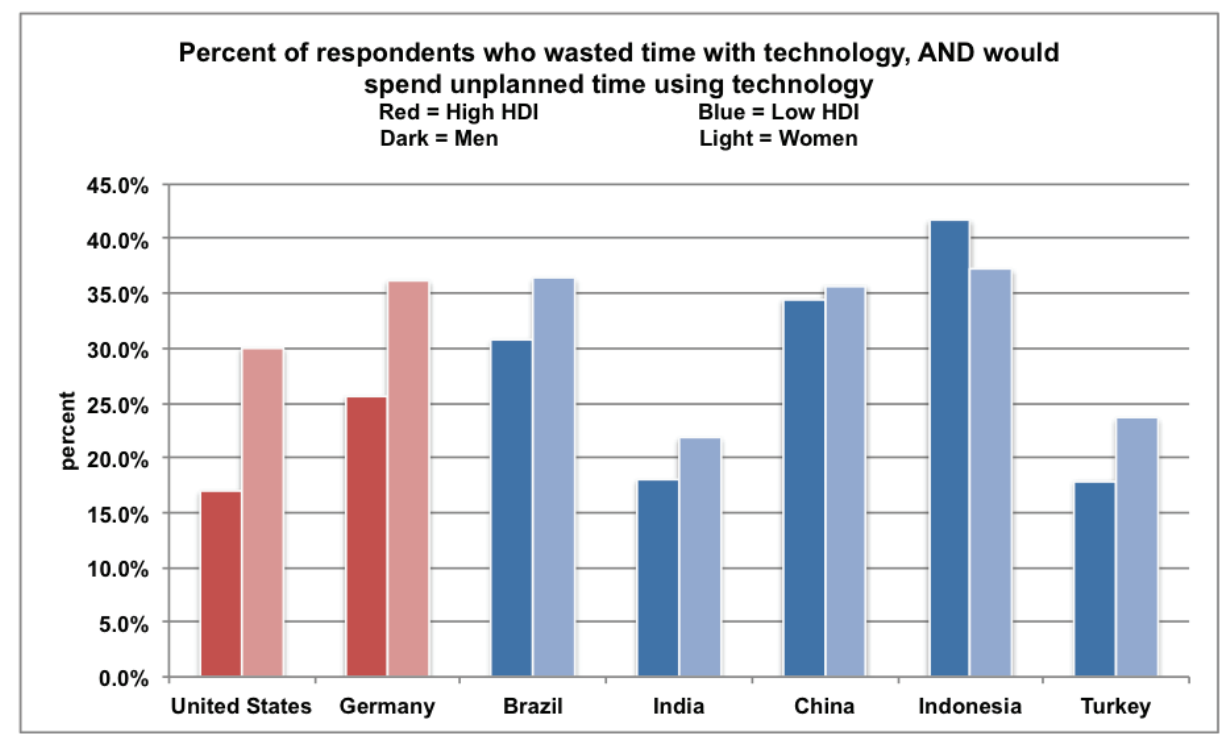

Figure 12.4: Percent of respondent who wasted time with technology, AND would spend unplanned time using technology

\subsubsection{Personal Meaning and the Optimal Experience}

As defined and described by Mihaly Csikszentmihalyi's concept of flow (Flow, The Psychology of Optimal Experience), optimal experiences happen when people 
deeply enjoy what they are doing - whether this be at work or in leisure. In fact, in earlier work, Csikszentmihalyi notes that "flow," most likely occurs when people are engaged in work, because of the inherent challenges involved. Building on this work in our study, we also find that meaning and engagement are the most important factors in terms of how people understand "quality." In other words, in defining an excellent restaurant meal, individuals would talk about the people around the table and the opportunity to talk with them about topics that matter, rather than the food, service, lighting, or general ambiance. Similarly, in terms of what makes one pair of shoes better than another, individuals would comment on whether the shoes would last a long time (that they were made well) or the experience that led the person to acquiring the shoes (maybe they purchased them with their best friend, or a pair of shoes were bought in preparation for a family trip). Price and convenience are rarely mentioned as indicators or criteria of quality.

Individuals in both our interviews and surveys described experiences in which they can deeply connect with others as "optimal" or "high quality" experiences. Furthermore, though we had predicted at the outset that individuals may define or make judgments about quality in terms of external criteria (e.g. price, status, etc.), we learned that in fact objects are only "high quality," if they are durable (last a long time) and if they represent a piece of someone's identity (either the person who owns the object or the person who gives the object). Individuals also explained that the time that goes into creating products and experiences can imbue them with meaning and characteristics that make them high quality in many individuals' eyes. In other words, the care and patience expressed by a barista in a coffee shop (even though the coffee may take longer to get) or a handmade card or a hand-written note (even though it may not be letter-perfect), are more important than a final product that might be flawless, but generated by a robot or someone unknown to the client.

Moreover, interviewees explained that they evaluate an object, in part, by considering whether the item helps them spend time in ways that are personally rewarding. Interview participants clarified that handmade goods are better because they reflect time investment. We noted with curiosity individuals' explanations that computers, cameras, and mobile devices are not deemed high quality just because of their reliability and features, but also because they help to maintain personal relationships, enable hobbies, capture and save time for special life moments. A young man who works in the music industry remarks: "Quality objects are things that I've been able to get enjoyment out of, that's what makes them quality, they play an important role in who I am.”

However, it is important to note that though many individuals believe in the value of doing things "by hand," they don't always care about the tools they use for such endeavors. In plain terms, individuals are not particular about the kind of writing implements they use - nearly two thirds of our survey sample responded that they do not believe that a higher quality tool (such as a pen or pencil) leads to a higher quality end product (such as a handwritten note). The quality is judged not on the tool or 
on the overall aesthetics of the product, but instead, the time and care expressed in creating something by hand and the enjoyment in creating it. These data indicate that people care more about the process than the product: the act of writing a note and the time the act represents, is most important.

Similarly, survey respondents also reported a preference for using "traditional" means to carry out tasks, rather than the digital alternatives (reading a book as opposed to using an e-reader, seeing a painting in a gallery as opposed to viewing it on the Internet). Interestingly, we found a relationship between the level of development within a country and individuals' preference for engaging with digital or traditional media: countries with lower HDI were more likely to want to engage with digital media as opposed to traditional media. This includes the need to write (by hand) as opposed to type (on a computer)-though nearly $60 \%$ of individuals surveyed in the United States, and 40\% in Germany and Indonesia, report regularly feeling the need to use a writing implement, fewer than $20 \%$ of respondents in Brazil, China, India, and Turkey report ever feeling the need to write by hand, as opposed to using a computer. Perhaps those who live in countries with less access to technology, value it more.

\subsubsection{Prioritizing Quality in Everyday Life}

To help individuals not only read about our findings, but also make use of them, we have embarked on two major applications for these learnings in two professional spheres, business and education. To promote the idea of "time well spent," we have started by working with a large global company (Faber-Castell) to encourage "time well spent" within and beyond their company through the development of an award focused on these ideas. In order to do this, we conducted a series of interviews with Faber-Castell employees, representing various high-level positions and markets around the world. Second, we developed a course for educators which encourages reflection about their understandings of quality and its implications for teaching and learning.

\subsubsection{Time Well Spent Award}

Interestingly, Faber-Castell hoped to find a way to incorporate the concept of "time well spent" both internally (within the company) and externally (with clients and customers). To learn about some of day-to-day activities within the company and how "time well spent" most resonated with employees, we conducted interviews to learn more about how this concept was applicable to the Faber-Castell workplace. Specifically, we were interested in what company employees consider to be "time well spent" in their work and personal lives, and how they believed the idea of "time well spent" is communicated to customers and consumers of Faber-Castell's products. Our inter- 
viewees ( $\mathrm{n}=27$ ) were universally receptive to the idea of "time well spent," and almost unanimously believed that it is a concept that should be on the minds of all FaberCastell employees.

In general, we learned that employees believe that Faber-Castell is a company that produces high-quality products and that cares about its employees. Employees almost unanimously believe in the company's mission to produce the highest-quality offering in its space, and believe that producing a quality product is top priority. The understandings we gathered from these interviews gave us the background necessary to work with Faber Castell on a new initiative to encourage "time well spent" both internally and externally-to help employees experience "time well spent" at work and ultimately, to encourage customers and clients to spend time well using their products (writing implements, art supplies, and cosmetics).

We are currently working on a Time Well Spent Award, which will be given to selected Faber-Castell employees who carry out projects that help others in their market, department, or office to spend their time "well." Through written materials describing the award, we will encourage project ideas to be varied-for example, some projects may address issues of human resources, marketing, or general administration-but all of them will be focused on the concept of "time well spent." We have developed a set of criteria for this award which describe categories to evaluate the project, including 1) working with others (e.g. collaboration, building and sustaining relationships); 2) lasting design (e.g. replicability, eco-sustainability); 3) cognitive inputs (e.g. reflection, creativity and innovation); and 4) quality (e.g. excellence and social responsibility). The criteria have been developed to be fairly flexible and brief in order to be translated into multiple languages and in order to allow for creativity. We hope that through the award program we will encourage new, innovative thinking about how to instill time well spent within the company and beyond.

\subsubsection{Quality Course}

Additionally, we have sought out the opportunity to apply our findings in the classroom. At Project Zero, our research group housed at the Harvard Graduate School of Education, we host an annual conference each July. Educators from around the world attend this gathering to learn about Project Zero research and its implications in the classroom. We believe that many of the findings from our Quality Study are relevant to teaching and learning in the $21^{\text {st }}$ century, and welcomed the chance to work with educators in this setting. We have now taught this course twice.

The goals of the course were for participants to explore their own definitions of "quality" and to unpack what this term means to them personally and professionally. Interestingly, throughout the course, we validated many of the findings from individuals all around the world - quality is most important in terms of time, and decisions about how to use time wisely (rather than to waste it, or just let it pass) are 
paramount. Spending time with family and friends-being around those for whom we care most - is much more important than spending time with technology or running errands. With this in mind, we asked participants about how we can ensure that students experience "quality" learning and teaching in school and also asked them to think further about how they define "quality" learning and teaching.

During this two and a half hour "mini course" we addressed four essential questions: 1) What is quality? 2) Why is quality important? 3) Who decides what quality is? and 4) How can we encourage quality? We explored these questions through a combination of presentation, hands-on activities, and discussion. Participants grappled with some of the same survey questions used in our research (to deepen their understandings of our research and to begin conversation about an otherwise overly-broad topic). Separating into pairs, they interviewed one another using questions also used in our research, asking them to consider how they spend their time, their judgments about their work and quality, and their preconceptions about the word "quality."

As part of our course, participants also worked with our (currently unpublished) text entitled Quality Through the Ages. The book is a compilation of 45 examples of quality over time-spanning some of the earliest inventions (e.g. clay and painting) to modern day monuments, professions, and other examples and spheres of quality (e.g. Shakespeare, tracking of time, the Internet). All of the participants read a short essay discussing the Olympics. We initially chose this particular essay because we thought that it brought up interesting issues for teachers and because it was timely (during our first teaching of the course, the Summer Olympics were just about to be held in London); during subsequent sessions we have returned to this essay because it inspires excellent conversation and enables educators from many diverse cultures to discuss their particular perspectives, for example, what excellence, such as the Olympics, means in their own setting. Specifically, we addressed important topics such as standards of quality in the classroom in terms of how to judge individual work and group work and whether it should be subjective or objective and how encouragement toward high quality can lead to too much pressure. Additionally, questions were raised about how to determine when standards of quality are unrealistic, and whether educators' definitions of quality are value-dependent. Educators also discussed some of the factors that impact quality work in the classroom, asking themselves how to encourage young people to work hard for intrinsic (learning for its own sake) rather than extrinsic rewards, e.g. winning awards, earning high marks and getting accepted to prestigious colleges and universities).

Interestingly, the issue of time was once again present in the discussion that emerged. In the classroom, knowing how to judge time is a crucial skill students need to learn. When is a paper "done?" When is it time to move on to the next assignment? Furthermore, the relationship of quality and balance proved a very useful concept for teachers. Balance related to many aspects of the course-how to strive for quality work and at the same time keep balance in our personal lives, how to balance the double-edged sword of technology use (it helps people be more efficient, but is also 
labeled as a waste of time), and how to encourage deep passion and "flow" in work, but not lose sight of the ultimate goal.

\subsection{Conclusion}

Though quality and quality time are regular and significant themes in public discourse, our study revealed interesting findings about the importance of "time well spent" and how time is paramount in individuals' understanding of quality, especially as it relates to both objects and services. Individuals across the globe have mixed feelings about the relationship between technology and time-while they know that technology can save time, they are conflicted about whether technology is "time well spent" or a time waste. In addition, though they value the efficiency technology provides, individuals most often prefer and appreciate the experience of carrying out daily tasks with non-digital means (such as writing thank you notes by hand, reading a tangible book). As discussed earlier, our research has also uncovered potentially important differences across countries and cultures regarding these findings. Based on this research and the applications we have developed, we conclude with a series of suggestions about how individuals can bring about optimal experiences in both their personal and professional lives.

Place the highest value on your use of time: As described in the research findings above, in general, individuals believe that time is the most important aspect of quality. As one interview participant commented: “Time well spent is...a scarce, fixed resource. How are you investing your time? It's the same way that people think about money. Twenty-four hours in the day, that's all you get...I don't think it takes a lot of explanation.” In other words, treat time as precious (we can't earn more of it), be careful not to "waste time" when possible, and reflect upon the kinds of activities that bring deep enjoyment to help spend time wisely.

Plan well; it takes time to decide how to use time: In our survey, we asked participants to think about what they might do if they had free, unplanned time, for example, if a half-a-day commitment was cancelled with short notice. Most individuals reported that they would not spend this "free" time engaged with activities that they had earlier (in the survey) indicated as "time well spent” (e.g. being with friends and family, hobbies, learning something new). In other words, individuals need time to plan and think clearly about how to use their time in advance of unplanned time. Choosing how to spend time "last minute," may lead to activities and tasks that people do not value.

Blend the use of on-line and on-line for activities and connections: Technology is a double-edged sword. It can be seductive and superficially fun, and sometimes considered to be "time well spent," but it is also a waste of time. In other words, using technology for efficiency, productiveness, and accessibility (connecting with people around the world) improves our lives greatly, but getting caught spending hours on 
Facebook, Twitter, or just "surfing the Internet," does not help create optimal experiences. Furthermore, as many of our interview participants explained, technology does not replace in-person, face-to-face experiences. Seeing a beautiful painting on the Internet is not the same as travelling to see the same painting in a museum. Reading a book by physically turning each page may bring more enjoyment than touching an arrow on an e-reader. Our quality of life is improved by technology, but at the same time, technology does not (and should not) replace the meaning and enjoyment of doing things "by hand."

Invest your best efforts, not just money: Individuals receive more pleasure from results when it is clear that time was invested in the product. Time indicates care. Individuals told us that they would rather stand in a long line to get coffee made by a barista who knows them well, rather than go somewhere else that might be quicker (and less expensive). Individuals would also rather go out of their way to pick out fruits and vegetables from an organic farm, rather than a large market, because they believe the food was handled and managed with care. These same individuals also told us that their family would value the meal more if they knew that someone went out of their way for the food brought to the table. Though money is helpful in many ways, and gives individuals access and security to many things in life, individuals derive meaning from objects, services, and work that express effort, care, and time.

Pursue and cherish quality, not mere quantity: Though quality and quantity are different concepts, in modern society they can be easily confused, and sometimes inextricably linked. Public perception is that quality reflects quantity or that quantity is an indicator of quality-high quality items are more expensive, things that take less time are better, status is attained when fewer people have access. However, throughout the process of our research, it is clear that people around the world do not seem to pursue quantity (or think of the meaningfulness of their lives in terms of this), individuals want to experience quality-mostly in terms of time well spent. Simply put, as Franklin stated many years ago, we should focus on the "quality," not the "quantity" of life.

Ultimately, these lessons bring us back to the opening line of this chapter, and a final life lesson: "Wish not so much to live long as to live well."

\section{References}

Csikszentmihalyi, M. \& Rochberg-Halton, E. (1981). The meaning of things: Domestic symbols and the self. Cambridge: Cambridge University Press.

deGraaf, J. (Ed.) (2003). Take back your time: Fighting overwork and time poverty in America. San Francisco, CA: Berrett-Koehler.

Economist: Schumpeter. (2013, August 17). In praise of laziness: Business people would be better off if they did less and thought more. The Economist.

Gabriel, Y. \& Lang, T. (1995). The unmanageable consumer: Contemporary consumption and its fragmentations. London: Sage Publications. 
Garvin, D. (1984, Fall). What does “Product Quality” really mean? Sloan Management Review, 26 (1), 25-43.

Gino, F. \& Mogilner C. (2013). Time, Money, and Morality. Psychological Science. Retrieved from http://pss.sagepub.com/content/early/2013/0956797613506438

Kasser, T., \& Sheldon, K. (2009). Time affluence as a parth toward personal happiness and ethical business practice: Empirical evidence from four studies. Journal of Business Ethics, 84, 243-255.

Kingwell, M. (2013, March 25). The Barbed Gift of Leisure. The Chronicle of Higher Education.

Masuda, A. D., \& Sortheix, F. M. (2012). Work-Family Values, Priority Goals and Life Satisfaction: A Seven Year Follow-up of MBA Students. Journal of Happiness Studies, 13(6), 1131-1144.

Mehl, M. R., Vazire, S., Holleran, S. E., \& Clark, C. S. (2010). Eavesdropping on Happiness: Well-Being is Related to Having Less Small Talk and More Substantive Conversations. Psychological Science, 21(4), 539-541.

Mezzich, J. E., Cohen, N. L., Ruiperez, M. A., Banzato, C. E., \& Zapata-Vega, M. I. (2011). The Multicultural Quality of Life Index: presentation and validation. Journal of Evaluation in Clinical Practice, 17, 357-364.

Mogilner, C., Chance, Z., \& Norton, M. I. (2012). Giving Time Gives You Time. Psychological Science, 23(10), 1233-1238.

Mulgan, G. (2013). Capitalism's Generative Ideas. The Locust and the Bee: Predators and Creators in Capitalism's Future (pp. 198-229). Princeton: Princeton University Press.

Parasuraman, A. Zeithamel, V.A. \& Berry, L. L. (1985, Fall). A conceptual model of service quality and its implications for future research. Journal of Marketing, 49, 41-50.

Parasuraman, A. Zeithamel, V.A. \& Berry, L. L. (1988). SERVQUAL: A multiple-item scale for measuring consumer perceptions of service quality. Journal of Retailing, 64 (1) 12-40.

Thompson, C. J. (2000). Postmodern consumer goals made easy!!! In S. Ratneshwar, D. G. Mick, \& C. Huffman, (Eds.) The why of consumption: Contemporary perspectives on consumer motives, goals and desires, (pp. 120-139). London: Routledge.

Turkle, S. (2007) What makes an object evocative? In S. Turkel, Evocative objects: Things we think with, (pp. 307-326). Cambridge, MA: MIT Press. 